\title{
Role of Behavioral Activation and Inhibition Systems in Symptoms of Major Depression Disorder Regarding the Mediating Role of Cognitive Bias
}

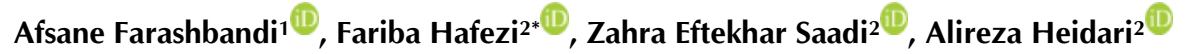 \\ 1 Ph.D. Student of General Psychology, Department of Psychology, Ahvaz Branch, Islamic Azad University, \\ Ahvaz, Iran \\ ${ }^{2}$ Associate Professor, Department of Psychology, Ahvaz Branch, Islamic Azad University, Ahvaz, Iran
}

*Corresponding author:

Fariba Hafezi, Department of Psychology, Ahvaz Branch, Islamic Azad University, Ahvaz, Iran

Email: febram315@yahoo.com

Received: 07 Jul. 2020 Accepted: 27 Oct. 2020 ePublished: 01 Nov. 2021

\begin{abstract}
Background and Objective: This study aimed to investigate the role of the behavioral activation and inhibition systems in symptoms of major depressive disorder, considering the mediating role of cognitive bias.

Materials and Methods: The statistical population of this descriptive-correlational research was all the students of Ahvaz Azad University, Ahvaz, Iran during the academic year 2015-16. In total, 300 students were selected using the multistage sampling method, and finally, 279 students participated in this research. Data were collected using the Behavioral Inhibition/Activation Systems Scale, the Dysfunctional Attitudes Scale, and the Beck Depression Inventory. The collected data were analyzed using multiple regression analysis, coefficient correlation, and structural equation modeling in SPSS (version 22), Lisrel (version 8.80), and Mplus (version 6.12) software.

Results: The results showed that cognitive bias mediated the influence of behavioral inhibition and activation systems on depressive disorder. Based on the findings, all of the direct and indirect effects of the model were significant. The model itself had a suitable index of fit, and cognitive bias significantly affected major depressive disorder symptoms. In the final model, the direct effect of the behavioral inhibition system was added to depression. Moreover, the indirect effect of the behavioral activation system on depression was not significant.
\end{abstract}

Conclusion: The proposed model had an acceptable fitness to the result and was an essential step in recognition of the significant factors of major depressive disorder. It can be useful as a model for designing stress management programs and decreasing major depression disorder.

Keywords: Behavioral activation system, Behavioral inhibition system, Cognitive bias, Depression disorder

\section{Background}

Major depressive disorder is one of the most specific mental disorders [1]. In the United States, $17 \%$ of adults have experienced at least one episode of major depression (MD) throughout their lives [2]. Symptoms of depression are related to disturbance of life in terms of interpersonal relationships, increased possibility of divorce, and decreased physical well-being. Moreover, depressive symptoms have a relationship with the rise in removal from office and poor job performance [3]. Some theorists and researchers argue that depression is associated with lower activity of reward systems. They consider depression the result of brain activities that lead to decreased tendencies towards pleasant stimuli [4].

Moreover, empirical evidence shows that individuals suffering from depression have hyperactive punishment systems that increase sensitivity in the face of annoying stimuli [5]. These reward and punishment systems are similar to behavioral activation (BAS) and behavioral inhibition systems (BIS) [6]. The BIS is defined as an incentive system related to sensitivity to punitive stimuli, elimination of reward, and new stimuli, while BAS is considered a motivational system that reacts to reward impulses or elimination of punishment [7].

Numerous studies have highlighted that intrinsic BAS/BIS inclinations emerge in personal styles and ultimately lead to emotional disorders [8]. In a study, BAS and BIS scores of patients with major depressive disorder were compared with those of the control group without depression. The results showed that lower scores on the BAS had a relationship with the severity of depression and anticipated more severe symptoms in the next eight months. They also indicated that depressed patients had higher scores on the BIS [9-11]. In their research, Gomez and Gomez [12] found that both BAS and BIS play a role in cognitive information 
processing and can predict bias in information processing. Kimbrel [13] also believes that BAS and BIS are types of bio-personality biases that lead to biased environmental information processing.

Cognitive theories of depression highlight the role of negative cognitive bias in the development and persistence of this disorder [14]. This cognitive bias is composed of the bias in memory [16], bias in attention [17], and negative interpretation bias in the form of a tendency to appraise ambiguous information in a negative way [15]. Although these cognitive biases are communal among most emotional disorders, their nature depends on the type of disorder [18]. Regarding depression, cognitive processing bias is subordinate to the underlying dysfunctional attitudes and beliefs [19]. The maladaptive beliefs and assumptions form a mental filter that will only allow information to be processed according to those beliefs [20,21].

The relationship between personality traits associated with BIS and cognitive bias can provide further evidence for the hypothesized model of this study. Compared to extroverts and emotionally stable individuals, neurotic introverts tend to bias attention towards negative environmental cues [22]. These findings provide evidence for the hypothesis that individuals with a higher level of BIS have a more significant cognitive bias towards negative information. Moreover, high levels of neuroticism are associated with a tendency towards recalling negative memories and thoughts about the self [23], maintaining negative beliefs about the self and world and one's ability to deal with it correctly, and obsessing with rumination and negative beliefs [24,25].

Overall, the direct relationships between the research variables have been supported by sufficient research; however, there is no significant study to investigate the impact of BAS and BIS on depression through cognitive bias mediation.

\section{Objectives}

This study aimed to evaluate this mediating role.

\section{Materials and Methods}

The statistical population of this descriptivecorrelational research was all the students of Ahvaz Azad University, Ahvaz, Iran during the academic year 2015-2016. The required sample size was based on previous research and the sample size was estimated based on the complexity of the hypothetical model. Some researchers, such as Marsh et al., have proposed the formula $\mathrm{n} \geq$ $50 r 2-450 r+1100$ for calculation of the minimum sample size where $\mathrm{n}$ is the sample size and $\mathrm{r}$ is the number of markers of each variable in each model. The p-value used for calculating the sample size was 0.05. Therefore, since in each of the hypothetical models of research, three markers have been considered for each latent variable, the minimum sample size for this study was 200 subjects. Hence, the total sample size used in this study was 300 individuals who were randomly selected using a multistage sampling method. They were selected from each of the four departments of humanities, technical and engineering education, primary and computer science, and agriculture and natural resources. Finally, 25 individuals from each field participated in this study. From this sample, the responses of 279 individuals were analyzed.

The inclusion criteria were meeting the DSM-IV diagnostic criteria for $\mathrm{MD}$, consulting for the first time to control therapeutic outcomes, and having good physical health. Patients were excluded from the study if they did not agree to participate in the study, had a current/past medical or psychiatric disorder in addition to depression, had a significant health problem, or had previously received psychiatric treatment. It should be mentioned that this article is ethically approved by Islamic Azad University, Ahvaz Branch (ethics code: IR.IAU.AHVAZ.REC.1397.037).

\section{Behavioral Inhibition/Activation Systems Scale}

The BAS/BIS Scale is a 24-item self-report questionnaire. Its BIS subscale includes seven items that assess the sensitivity of BIS in reaction to threat symptoms. Moreover, its BAS subscale contains 13 items that evaluate the sensitivity of the BAS. The BAS itself includes three subscales in this questionnaire, namely drive (4 items), reward responsiveness (5 items), and fun-seeking (4 items). The four additional items listed as cover scale items do not play a role in BAS/BIS assessment. The items are rated by the subject based on a four-point Likert scale. The internal consistency values of the BIS and BAS scales were 0.74 and 0.71 , respectively [26].

\section{Dysfunctional Attitudes Scale}

This scale was developed from its original 22-item version to be used in the Iranian clinical population, and its psychometric quality was also confirmed. The items assess the core beliefs of depressed individuals and their pathological attitudes toward the self, future, and others. The Dysfunctional Attitudes Scale is a 26-item self-report tool and is scored based on a seven-point Likert scale. Alpha Cronbach of this tool was 0.97, and its correlation with the original scale was 0.97. Its predictive validity through correlation with General Health Questionnaire-28 scores was 0.56 [27]. 
Beck Depression Inventory

It is a 13-item self-report tool that is used to measure the presence and severity of cognitive, emotional, and physical symptoms of depression over the past two weeks. Items are rated on a four-point Likert scale ranging from zero to three. The BDI-SF has revealed good psychometric properties and has been widely used to evaluate major depressive disorder symptoms. A Cronbach alpha of 0.84 has also been reported for its internal consistency [28]. The findings were analyzed using multiple regression analysis, coefficient correlation, and structural equation modeling. The data were analyzed in SPSS software (version 22), Lisrel (version 8.80), and Mplus (version 6.12) software.

\section{Results}

The sample consisted of $131(47 \%)$ males and $137(49.1 \%)$ females. Furthermore, 11 (3.9\%) individuals had not specified their gender. The mean ages of male and female participants were $22.99 \pm 2.18$ and $21.93 \pm 1.77$, respectively. Regarding their academic education, 187 (67\%), 79 (28.31\%), and $13(4.6 \%)$ individuals were undergraduate students, Master's students, and Ph.D. students, respectively. Regarding their field of study, 72 (28.31\%), 67 (24\%), 69 (24.73\%), and $71(25.44 \%)$ were studying humanities, engineering, basic science, and computer, respectively. Table 1 summarizes the descriptive indices of variables used in the study, namely minimum value, maximum value, mean, standard deviation, skewness, and kurtosis.

Table 5 tabulates the model fit indices in which cognitive bias played a mediating role between the effects of exogenous and endogenous variables. As can be seen, the values of the comparative fit index and Tucker-Lewis index indices are within the model fit range. In addition, the values obtained for the root mean square error of approximation and weighted root mean square residual indices are within the scope of the acceptable model fit. Therefore, it can be concluded that this hypothetical model has a proper fit.

TLI: Tucker-Lewis index, CFI: comparative fit index, RMR: root mean square residual, RMSEA: root mean square error of approximation

Table 6 summarizes the details regarding the direct paths of the hypothetical model with the mediation of cognitive bias. It includes nonstandard coefficient, standard coefficient, standard error, significance level, and the result of the division of the standard coefficient by the standard error which is the best index for comparing the disruption coefficients of a model.

BIS: Behavioral inhibition system, BAS: Behavioral activation system, CB: Cognitive bias

Table 1. Descriptive indices of research variables

\begin{tabular}{lcccc}
\hline & Mean & SD & Skewness & Kurtosis \\
\hline Behavioral inhibition system & 19.77 & 2.57 & 0.105 & 0.314 \\
Behavioral activation system & 38.28 & 3.24 & 0.362 & -0.517 \\
Cognitive bias & 58.91 & 17.80 & -0.154 & -0.793 \\
Major depression disorder & 7.40 & 6.51 & 1.10 & 0.437 \\
\hline
\end{tabular}

Table 2. Correlation Matrix of Research Variables

\begin{tabular}{|c|c|c|c|c|c|}
\hline & 1 & 2 & 3 & 4 & 5 \\
\hline Behavioral inhibition system & 1 & & & & \\
\hline Behavioral activation system & -0.102 & 1 & & & \\
\hline Cognitive bias & $0.398^{* *}$ & $-0.292 *$ & 1 & & \\
\hline Difficulty in emotion regulation & $0.267^{* *}$ & -0.131 & $0.430^{* *}$ & & \\
\hline Major depressive disorder & $0.272^{* *}$ & $-0.278^{* *}$ & $0.475^{* *}$ & $0.500^{* *}$ & 1 \\
\hline
\end{tabular}

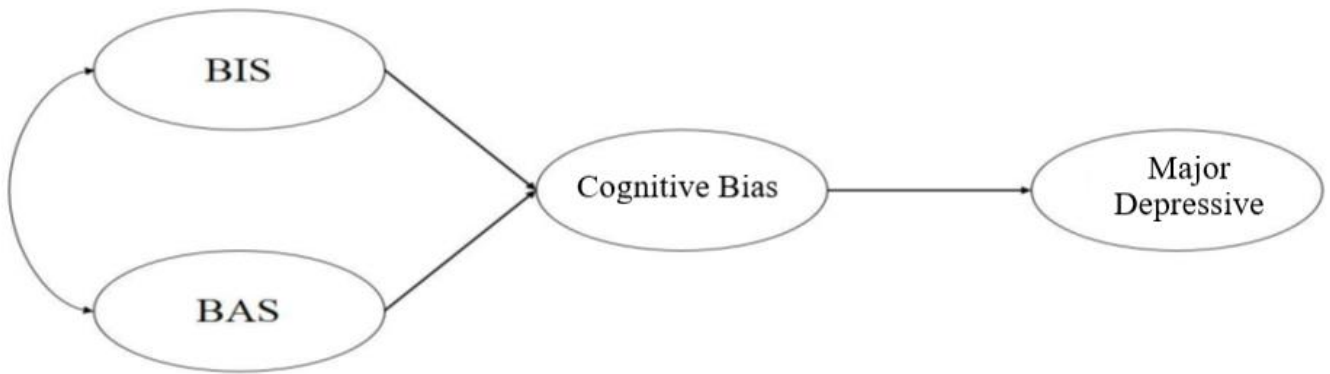

Figure 1. Hypothetical model of research with the mediating variable of cognitive bias. BIS: Behavioral inhibition system, BAS: Behavioral activation system 
Table 3. Indices of fit for the measurement model

\begin{tabular}{lccc}
\hline TLI & CFI & RMR & RMSEA \\
\hline 0.950 & 0.942 & 0.097 & 0.098 \\
\hline TLI: Tucker-Lewis index, CFI: comparative fit index, RMR: root mean square residual, RMSEA: root mean square error of approximation
\end{tabular}

Table 4. Standardized coefficient, standardized error, and the significance level of the observed variables

\begin{tabular}{|c|c|c|c|}
\hline & Standardized coefficient & Standardized error & Significance level \\
\hline \multicolumn{4}{|l|}{ Observed variables of BIS } \\
\hline BIS1 & 0.436 & 0.071 & 0.001 \\
\hline BIS2 & 0.640 & 0.119 & 0.001 \\
\hline BIS3 & 0.492 & 0.094 & 0.001 \\
\hline \multicolumn{4}{|l|}{ Observed variables of BAS } \\
\hline BAS1 & 0.749 & 0.121 & 0.001 \\
\hline BAS2 & 0.445 & 0.088 & 0.001 \\
\hline BAS3 & 0.586 & 0.119 & 0.001 \\
\hline \multicolumn{4}{|l|}{ Observed variables of Cognitive bias } \\
\hline CB1 & 0.720 & 0.038 & 0.001 \\
\hline CB2 & 0.722 & 0.041 & 0.001 \\
\hline CB3 & 0.854 & 0.044 & 0.001 \\
\hline \multicolumn{4}{|l|}{ Observed variables of MDD } \\
\hline MDD1 & 0.864 & 0.030 & 0.001 \\
\hline MDD2 & 0.990 & 0.023 & 0.001 \\
\hline MDD3 & 0.839 & 0.038 & 0.001 \\
\hline
\end{tabular}

BIS: Behavioral inhibition system, BAS: Behavioral activation system, CB: Cognitive bias, MDD: Major depressive disorder

In this study, the Bootstrap test was used to evaluate the mediating relationships. The path of BIS to major depressive disorder was mediated by cognitive bias with a standard coefficient of 0.692 which was significant at the level of $\mathrm{P}<0.01$. The path of BAS to major depressive disorder with the mediation of cognitive bias with a standard coefficient of -0.222 was significant at the level of $\mathrm{P}<0.05$.

\section{Discussion}

There is empirical support for the causative role of BIS and BAS in the development of emotional disorders, such as depression. However, the findings of a few studies have indicated the exact mechanism through which these biological factors affect conditions. Research about the factors that play a mediating role in the relationship between bio-personality factors and major depressive disorder will improve our understanding of the etiology of this disease and lead to more effective treatments. These findings are consistent with those of the studies performed by Abdolmanafi et al. [29] and Joormann and Gotlib [30]. Their results support the robust relationships among anger, depression, emotion regulation, and anger rumination.
This research sought to test the structural model in which cognitive bias mediated the influence of BIS and BAS on depressive disorder. Based on the findings of this study, all of the direct and indirect impacts presented in the model were significant, and the model itself had a proper index of fit. In the final model, the direct effect of BIS was added to depressive disorder; moreover, the indirect effect of BAS on depression was not significant.

In the research model in which cognitive bias played a mediating role, the direct effect of BIS and BAS on cognitive bias was significant. It should also be mentioned that the effect of BIS was more noticeable. The BIS is a system that has always sought to search for intrinsic and extrinsic threatening information in potentially threatening situations [31]. The BIS is considered the biopersonality basis of cognitive biases in individuals with emotional disorders. The result of this biased cognitive processing is the perception or interpretation of ambiguous and new terms and conditions in a threatening manner. This processing type per se results in constant fear and anxiety and avoidance of new experiences [32].

Both depressed and recovered participants obtained lower scores on the self-report scale than controls, suggesting a hypoactive BAS. This difference,

Table 5. Fit indices of the first structural model (with the mediation of cognitive bias)

\begin{tabular}{lcccc}
\hline TLI & CFI & RMR & RMSEA & Chi-Square \\
\hline 0.945 & 0.940 & 1.187 & 0.109 & 112.519 \\
\hline
\end{tabular}

Table 6. Indices of direct paths of the model (with mediation of cognitive bias)

\begin{tabular}{lcccc}
\hline Path & Non-standard coefficient & $\begin{array}{c}\text { Standard } \\
\text { coefficient }\end{array}$ & Standard error & Standard coefficient \\
\cline { 3 - 5 } BIS $\Leftrightarrow$ BAS & 0.012 & 0.074 & 0.082 & Standard error \\
BIS $\Rightarrow$ CB & 1.655 & 0.808 & 0.067 & -0.900 \\
BAS $\Rightarrow$ CB & -0.506 & -0.265 & 0.088 & 0.368 \\
\hline
\end{tabular}




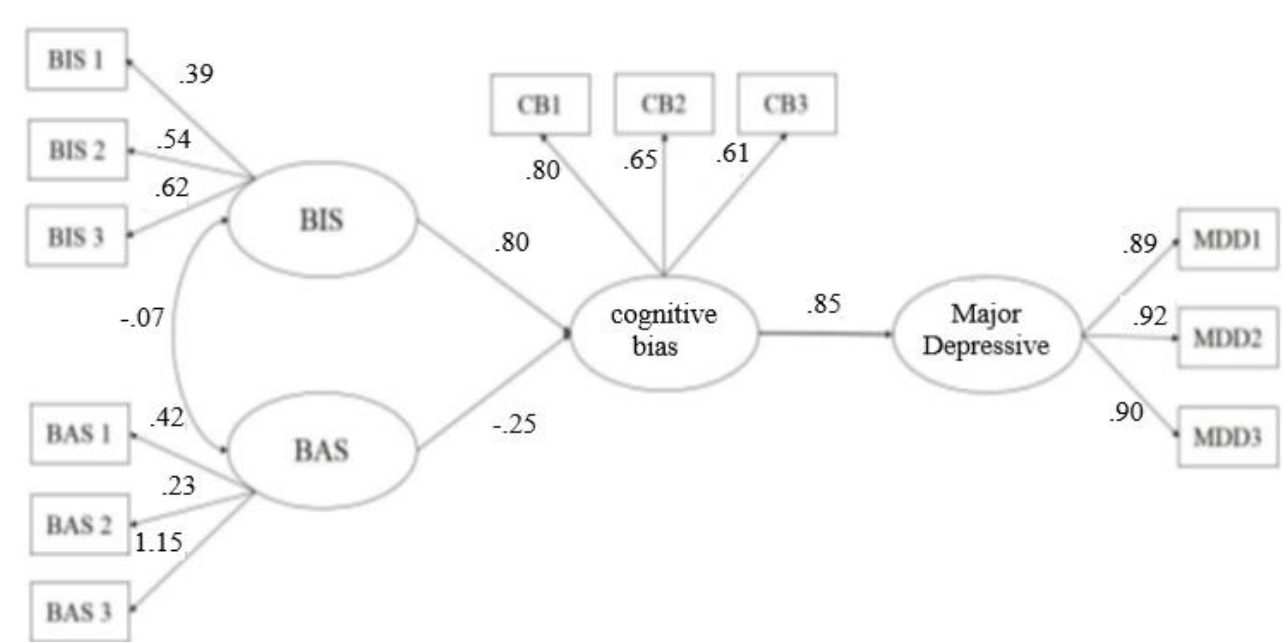

Figure 2. Structural Model of Research (with the mediation of cognitive bias). BIS: Behavioral inhibition system, BAS: Behavioral activation system, CB: Cognitive bias, MDD: Major depressive disorder

persisted after controlling the scores on scales of anxiety and depressive symptoms suggesting that it could not be explained just by the presence of such symptoms. Results of the present study are inconsistent with those reported by Johnson et al. [9] that suggested no relationship between BAS activity and depression among the general population. However, the findings of the present research were in line with those obtained by Kasch et al. [8]. They used different scales to assess BIS and BAS and found an underactive BAS in depressed participants that remained sfigure after the improvement of depressive symptoms. Therefore, it seems that the BAS-MD relationship in clinical samples is robust and independent of the used questionnaire.

A hypoactive BAS is associated with a higher threshold for presenting approach behavior in response to appetitive signals and a behavioral style characterized by a low emission of approach-related behaviors oriented towards the achievement of a reward. Therefore, subjects prone to depression would be those with a general low tendency to approach appetitive stimuli. At this point, it can be argued that behavioral therapy for depressive disorders based on the systematic increase of exposure to positive activities could base its effectiveness on reversing the usually observed pattern of poor approach behaviors. This intervention has shown promising results for the treatment of MD [11] and reduction of relapses [10].

In any case, these conclusions about behavioral therapy are purely speculative, and specific research on this issue should be conducted to reach any conclusions. Furthermore, prospective studies are also needed to clarify if BAS hypoactivity truly represents a trait-vulnerability marker as suggested by Kasch et al. [8] and our data or if it is a "scar" resulting from the experience of an MD episode.

People lead their attention towards positive or negative events to adapt to their previous experiences through information processing. Due to the negative bias in information processing, depressed people tend to perceive cues for sadness or dysphoria negatively [19] and interpret neutral or positive information negatively as well [21]. Negative bias in information processing is a feature of MD [24]. Dysfunctional schemas and consequently, dysfunctional attitudes lead people with MD towards failures in the evaluation of life events [20].

On the whole, these people experience more harmful and destructive emotions, such as anger, than nondepressed individuals. Simple and usual anger experiences cannot explain the quality and quantity of this emotion in depressed people. Results of this study showed that cognitive bias mechanisms are useful in this relationship. People use different coping strategies in the face of adverse events. These cognitive biases play a crucial role in the relationship between experiencing adverse life events and reporting depression and anxiety symptoms.

Studies about the relationship between BIS-based personality traits and cognitive bias provide additional evidence that supports the findings of this study. For example, Deribery and Red reported that neurotic introverts have a higher tendency for showing bias towards negative symptoms, in comparison with persistent extroverts. Since the neurotic introverts have higher BIS, and emotionally stable extroverts have lower BIS [13], these findings further support this hypothesis that BIS has a significant effect on cognitive bias. Moreover, higher levels of neuroticism are associated with a tendency towards frequent 
recalling of negative memories and thoughts about oneself, maintaining negative beliefs about the world and one's ability to deal with it, and rumination of and engagement with dysfunctional beliefs [24].

Furthermore, a study should be conducted on clinical or non-clinical populations to increase the generalizability of its findings. It is suggested that longitudinal studies be conducted to infer causal relationships and clarify the temporal sequence of research variables. It is better to employ different measurement methods rather than only selfassessment scales to represent research variables better. For example, the cognitive homework used to assess attentional bias and memory can evaluate cognitive biases. In future research, using different measurement methods, such as physiological and behavioral ones and self-rating instruments, can help better conceptualize variables and increase the validity of findings.

\section{Ethical Considerations}

All ethical principles were considered in this research; accordingly, the participants were informed about the purpose of the study and its stages. In addition, informed consent was obtained from the subjects. They were also assured of the confidentiality of their information. Moreover, the subjects were free to withdraw from the study. They were also informed that they would be provided with the results of the research.

\section{Funding/Support}

This research did not receive any specific grant from funding agencies in the public, commercial, or not-for-profit sectors.

\section{Authors' Contributions}

Conceptualization: Afsane Farashbandi; Methodology: Fariba Hafezi; Investigation: Zahra Eftekhar Saadi; Writing of the Original Draft: Alireza Heidari; Writing, Reviewing, and Editing: all authors; Funding Acquisition: all author; Resources: all authors; Supervision: Fariba Hafezi.

\section{Conflicts of Interest}

The authors declare that they have no conflicts of interest related to this study.

\section{Acknowledgments}

The authors would like to thank the participants who greatly cooperated with them in the research. [DOI:] [PMID] [PMCID]

\section{References}

1. Atadokht A, Majdy N. Comparison of brain/behavioral systems, cognitive emotion regulation, and cognitive failures in patients with schizophrenia disorder, major depression disorder, and normal individuals. Iranian Journal of Psychiatric Nursing. 2017; 5(4):8-18. [DOI:10.21859/ijpn05042]

2. Apazoglou K, Kıng AL, Cordera P, Aubry JM, Dayer A, Vuilleumier $\mathrm{P}$, et al. Rumination related activity in brain networks mediating attentional switching in euthymic bipolar patients. International Journal of Bipolar Disorders. 2019; 7(1):3-12. [DOI:10.1186/s40345-018-0137-5] [PMID] [PMCID]

3. Huh HJ, Kim KH, Lee HK, Chae JH. The relationship between childhood trauma and the severity of adulthood depression and anxiety symptoms in a clinical sample: the mediating role of cognitive emotion regulation strategies. Journal of Affective Disorders. 2017; 213:44-50. [DOI:10.1016/j.jad.2017.02.009] [PMID]
4. Barrys-Loscertales A, Meseguer V, Sanjuбn A, Belloch V, Parcet MA, Torrubia $R$, et al. Behavioral inhibition system activity is associated with increased amygdala and hippocampal gray matter volume: a voxel-based morphometry study. Neuroimage. 2006; 33(3):1011-5. [DOI:10.1016/j.neuroimage.2006.07.025] [PMID]

5. Shank DB, DeSanti A. Attributions of morality and mind to artificial intelligence after real-world moral violations. Computers in Human Behavior. 2018; 86:401-11. [DOI:10.1016/j.chb.2018.05.014]

6. Jones KC, Welton SR, Oliver TC, Thoburn JW. Mindfulness, spousal attachment, and marital satisfaction: A mediated model. The Family Journal. 2011; 19(4):357-61. [DOI:10.1177/1066480711417234]

7. Merens W, Van der Does AW, Spinhoven P. The effects of serotonin manipulations on emotional information processing and mood. Journal of Affective Disorders. 2007; 103(1-3):4362. [DOI:10.1016/j.jad.2007.01.032] [PMID]

8. Johnson SL, Turner RJ, Iwata N. BIS/BAS levels and psychiatric disorder: an epidemiological study. Journal of Psychopathology and Behavioral Assessment. 2003; 25(1):25-36. [DOI:10.1023/A:1022247919288]

9. Kasch KL, Rottenberg J, Arnow BA, Gotlib IH. Behavioral activation and inhibition systems and the severity and course of depression. Journal of Abnormal Psychology. 2002; 111(4):589-97. [DOI:10.1037//0021-843x.111.4.589] [PMID]

10. Fayazi M, Hasani J. Structural relations between brainbehavioral systems, social anxiety, depression, and internet addiction: with regard to revised reinforcement sensitivity theory (r-RST). Computers in Human Behavior. 2017; 72:441-8. [DOI:10.1016/j.chb.2017.02.068]

11. Li Y, Xu Y, Chen Z. Effects of the behavioral inhibition system (BIS), behavioral activation system (BAS), and emotion regulation on depression: a one-year follow-up study in Chinese adolescents. Psychiatry Research. 2015; 230(2):28793. [DOI:10.1016/j.psychres.2015.09.007] [PMID]

12. Haney JM, Hardie L. Psychotherapeutic considerations for working with betrayed spouses: a four-task recovery model. Australian and New Zealand Journal of Family Therapy. 2014; 35(4):401-13. [DOI:10.1002/anzf.1073]

13. Starratt VG, Weekes-Shackelford V, Shackelford TK. Mate value both positively and negatively predicts intentions to commit an infidelity. Personality and Individual Differences. 2017; 104:18-22. [DOI:10.1016/j.paid.2016.07.028]

14. Jiang Z, Zhao X. Brain behavioral systems, self-control and problematic mobile phone use: the moderating role of gender and history of use. Personality and Individual Differences. 2017; 106:111-6. [DOI:10.1016/j.paid.2016.10.036]

15. Alavi Lavasani AA, Ahmadi Thouri M. Association between maltreatment in childhood and emotional divorce: mediating role of attachment styles, early maladaptive schemas and difficulty in emotional regulation. Journal of Health Promotion Management. 2017; 6(4):49-58.

16. Pines AM, Neal MB, Hammer LB, Icekson T. Job burnout and couple burnout in dual-earner couples in the sandwiched generation. Social Psychology Quarterly. 2011; 74(4):361-86. [DOI:10.1177/0190272511422452]

17. Leeker O, Carlozzi A. Effects of sex, sexual orientation, infidelity expectations, and love on distress related to emotional and sexual infidelity. Journal of Marital and Family Therapy. 2014; 40(1):68-91. [DOI:10.1111/j.17520606.2012.00331.x] [PMID]

18. Hembling I Andrinopoulos K. Evidence of increased $\mathrm{STI} / \mathrm{HIV}$-related risk behavior among male perpetrators of intimate partner violence in Guatemala: results from a national survey. AIDS Care. 2014; 26(11):1411-8 [DOI:10.1080/09540121.2014.913766] [PMID]

19. Conroy AA. Marital infidelity and intimate partner violence in rural malawi: a dyadic investigation. Archives of Sexual Behavior. 2014; 43(7):1303-14. [DOI:10.1007/s10508-0140306-2] [PMID] [PMCID]

20. Barzoki MH, Tavakoll M, Burrage H. Rational-emotional 'divorce' in Iran. Applied Research in Quality of Life. 2015; 10(1):107-22. [DOI:10.1007/s11482-014-9303-9]

21. Brassiolo P. Domestic violence and divorce law: when divorce threats become credible. Journal of Labor Economics. 2016; 34(2):443-77. [DOI:10.1086/683666]

22. King DB, DeLongis A. When couples disconnect: 
Rumination and withdrawal as maladaptive responses to everyday stress. Journal of Family Psychology. 2014; 28(4):460-9. [DOI:10.1037/a0037160] [PMID]

23. Fischer TD, Smout MF, Delfabbro $\mathrm{PH}$. The relationship between psychological flexibility, early maladaptive schemas, perceived parenting and psychopathology. Journal of Contextual Behavioral Science. 2016; 5(3):169-77. [DOI:10.1016/j.jcbs.2016.06.002]

24. Flink $N$, Lehto SM, Koivumaa-Honkanen $H$, Viinam $\Delta$ ki $H$, Ruusunen A, Valkonen-Korhonen $M$, et al. Early maladaptive schemas and suicidal ideation in depressed patients. The European Journal of Psychiatry. 2017; 31(3):87-92. [DOI:10.1016/j.ejpsy.2017.07.001]

25. Balderrama-Durbin C, Stanton K, Snyder DK, Cigrang JA, Talcott GW, Slep AM, et al. The risk for marital infidelity across a year-long deployment. Journal of Family Psychology. 2017; 31(5):629-34. [DOI:10.1037/fam0000281]

26. L6ng A. Machiavellianism and early maladaptive schemas in adolescents. Personality and Individual Differences.
2015; 87:162-5. [DOI:10.1016/j.paid.2015.07.039]

27. Nash B, Chapman NA. Building a culture of caring: Lessons learned from managing professional expectations while navigating the emotional upheaval of divorce. Journal of Psychotherapy Integration. 2019; 29(2):95. [DOI:10.1037/int0000146]

28. Olugbenga AJ. Patterns and causes of marital conflict among staff of selected universities in southwest Nigeria. Advances in Social Sciences Research Journal. 2018; 5(8):4988. [DOI:10.14738/assri.58.4988]

29. Abdolmanafi A, Besharat MA, Farahani H, Khodaii MR. The moderating role of locus of control on the relationship between anger and depression in patients with major depression disorder. Procedia-Social and Behavioral Sciences. 2011; 30:297-301. [DOI:10.1016/j.sbspro.2011.10.059]

30. Joormann J, Gotlib $\mathrm{IH}$. Emotion regulation in depression: relation to cognitive inhibition. Cognition and Emotion. 2010; 24(2):281-98. [DOI:10.1080/02699930903407948] 